\title{
A Metabolic Landscape for Maintaining Retina Integrity and Function
}

\author{
Filipe O. Viegas ${ }^{1,2}$ and Stephan C. F. Neuhauss ${ }^{1 *}$ \\ 'Department of Molecular Life Sciences, University of Zurich, Zurich, Switzerland, ${ }^{2}$ Life Science Zurich Graduate School, \\ Ph.D. Program in Molecular Life Sciences, Zurich, Switzerland
}

Neurons have high metabolic demands that are almost exclusively met by glucose supplied from the bloodstream. Glucose is utilized in complex metabolic interactions between neurons and glia cells, described by the astrocyte-neuron lactate shuttle (ANLS) hypothesis. The neural retina faces similar energy demands to the rest of the brain, with additional high anabolic needs to support continuous renewal of photoreceptor outer segments. This demand is met by a fascinating variation of the ANLS in which photoreceptors are the central part of a metabolic landscape, using glucose and supplying surrounding cells with metabolic intermediates. In this review we summarize recent evidence on how neurons, in particular photoreceptors, meet their energy and biosynthetic requirements by comprising a metabolic landscape of interdependent cells.

\section{OPEN ACCESS}

Edited by:

Daniele Dell'Orco,

University of Verona, Italy

Reviewed by:

Pierre J. Magistretti, King Abdullah University of Science and Technology, Saudi Arabia James Hurley,

University of Washington, United States

*Correspondence:

Stephan C. F. Neuhauss stephan.neuhauss@m/s.uzh.ch

Received: 19 January 2021 Accepted: 22 March 2021 Published: 15 April 2021

Citation: Viegas FO and Neuhauss SCF (2021) A Metabolic Landscape for Maintaining Retina Integrity and Function.

Front. Mol. Neurosci. 14:656000. doi: 10.3389/fnmol.2021.656000
Keywords: metabolism, retina, brain, photoreceptor, glucose metabolism

\section{INTRODUCTION TO GLUCOSE METABOLISM}

Although cells fulfill a plethora of different functions, their functionality ultimately hinges upon the cell's ability to generate energy for survival and cell division. The energy currency of cells is ATP, enabling thermodynamically unfavorable biological reactions. Depending on the availability of resources, unicellular organisms are able to shift their metabolism between consuming nutrients to produce energy (catabolism) and using this energy to produce the building blocks of a new cell (anabolism). Multicellular organisms experience similar demand-driven shifts in metabolism. These are particularly prominent in dividing tissue but also occur in postmitotic cells, which can shift toward anabolic states (Vander Heiden et al., 2009; Lunt and Vander Heiden, 2011).

Catabolic reactions essentially consist of breaking-down molecules into smaller components that are that are oxidized to either provide energy or create building blocks for anabolic reactions. The

Abbreviations: ADP, adenosine diphosphate; AMD, age-related macular degeneration; ANLS, astrocyte-neuron lactate shuttle; AP, action potential; ATP, adenosine triphosphate; BRB, blood-retina barrier; BSG, basigin; CNS, central nervous system; DHAP, dihydroxyacetone phosphate; EAAT, excitatory amino acid transporter; FAD, flavin adenine dinucleotide; FADH, flavin adenine dinucleotide hydroquinone; G6P, glucose-6-phosphate; GCL, ganglion cell layer; GLUT, glucose transporter; HK, hexokinase; INL, inner nuclear layer; IPL, inner plexiform layer; LDH, lactate dehydrogenase; MCT, monocarboxylate transporter; MGC, müller glia cell; NAD, nicotinamide adenine dinucleotide; NADH, nicotinamide adenine dinucleotide hydrogen; NADP, nicotinamide adenine dinucleotide phosphate; NADPH, nicotinamide adenine dinucleotide phosphate hydrogen; ONL, outer nuclear layer; OPL, outer plexiform layer; OS, outer segment; OXPHOS, oxidative phosphorylation; PDH, pyruvate dehydrogenase; PFK, phosphofructokinase; PhR, photoreceptor; PKM1/M2, pyruvate kinase isozyme M1/M2; PPP, pentose phosphate pathway; RdCVF, rod-derived cone viability factor; ROS, reactive oxygen species; RP, retinitis pigmentosa; RPE, retinal pigment epithelium; TCA, tricarboxylic acid. 
most widely used carbon source is glucose, which is eventually catabolized to gain ATP, but can also provide crucial precursors for biosynthetic reactions. There are two major routes of glucose metabolism: Either, glucose feeds into glycolysis, where it is oxidized and becomes pyruvate, providing ATP and metabolic intermediates; or alternatively, glucose is shuttled from glycolysis into the pentose phosphate pathway for nucleic acid synthesis and the formation of NADPH (Figure 1).

Glycolysis is carried out by a set of cytoplasmic enzymes ultimately yielding pyruvate, catalyzing the phosphorylation of two ADP to ATP and causing the reduction of two $\mathrm{NAD}^{+}$to $\mathrm{NADH}$ (Figure 1). In the presence of oxygen, pyruvate is oxidized to Acetyl-CoA and enters the mitochondrial tricarboxylic acid (TCA) cycle (also known as Krebs cycle or citric acid cycle). In the TCA cycle there are a series of oxidative reactions resulting in the generation of reductive power in the form of $\mathrm{NADH}$ and $\mathrm{FADH}_{2}$ (Figure 1). The electron transport chain in the membrane of the mitochondria then uses this reductive power to generate ATP in a process termed oxidative phosphorylation (OXPHOS). This is the most profitable component of energy metabolism since one glucose molecule generates up to 36 molecules of ATP (Figure 1).

When oxygen is limited, cells shift pyruvate away from the TCA and OXPHOS by reducing it to lactate in a process termed anaerobic respiration. Under oxygen-deprived conditions, $\mathrm{NADH}$ can no longer be reduced to $\mathrm{NAD}^{+}$. Since $\mathrm{NAD}^{+}$is still required as an electron accepter, pyruvate is reduced to lactate, consuming $\mathrm{NADH}$ and replenishing the pool of $\mathrm{NAD}^{+}$in the process. Under special circumstances, anaerobic respiration can also occur when oxygen is abundantly available, as will be detailed below.

In addition to being the backbone of energy metabolism, glycolysis and the TCA cycle also provide essential precursors for biosynthetic pathways. Several glycolytic intermediates contribute toward amino acid biosynthesis, nucleotide biosynthesis, complex carbohydrate biosynthesis, as well as lipid biosynthesis (Figure 1). The first step of glycolysis converts glucose into Glucose-6-phosphate (G6P) by hexokinase (HK) enzymes. G6P can either be ultimately metabolized to pyruvate, or be diverted to the pentose phosphate pathway (PPP), which provides the building blocks for nucleotides as well as replenishing NADPH (Figure 1). The other noteworthy biosynthetic contribution of glycolysis is generating precursors for phospholipid synthesis. The glycolytic intermediate dihydroxyacetone phosphate (DHAP) can be oxidized to glycerol-3-phosphate, which is an essential precursor of diacyl glycerophospholipids and glycerophospholipids, structural components of all biological membranes. Similarly to glycolysis, the TCA cycle provides important precursors for lipid and amino acid biosynthesis (Figure 1).

While the catabolic and anabolic roles of the described pathways are essential for all cells, variations serving different metabolic requirements exist. This is particularly true for neurons.

Due to their particular physiology and function, neurons are energetically demanding and rely almost exclusively on glucose for the energy they need (Ames, 2000; Dienel, 2019). The particular importance of neuronal energy metabolism stems from the steep energy requirements of neuronal signaling. Neurons function by transiently changing their membrane potential, which in turn poses a high metabolic burden on regaining resting potential through the activity of ATP-dependent ion pumps.

Next, we will focus on energy metabolism of neurons in the brain and the retina, and attempt to present a cohesive model of retinal energy metabolism.

\section{BRAIN ENERGY METABOLISM}

The brain is among the most metabolically active organs and it relies almost exclusively on glucose as its energy source (Howarth et al., 2012; Magistretti and Allaman, 2015; Ashrafi and Ryan, 2017; Dienel, 2019). It accounts for about 20\% of total body oxygen consumption and $25 \%$ of total glucose usage while comprising only around $2 \%$ of total body weight (Sokoloff, 1977; Mink et al., 1981). Brain function relies on the partnership between neurons, glia and vasculature to meet such large metabolic requirements (Figure 2). Astrocytes are the most abundant and most relevant glia cells for brain energy metabolism (Barros et al., 2018; Jha and Morrison, 2018). They play several roles in maintaining proper brain function, some of which are directly linked to energy metabolism (Bélanger et al., 2011; Magistretti and Allaman, 2015). Astrocytes mediate the link between neuronal energy needs and circulating glucose by directly contacting and surrounding blood vessels with projections (endfeet), as well as regulating blood supply relative to neuronal demand (Oberheim et al., 2006; Gordon et al., 2008) by contributing to vasomotor responses (Attwell et al., 2010).

Astrocytes act as the main entry point of glucose into the brain via glucose transporters (GLUTs) expressed at both endothelium and astrocyte endfeet (Figure 2). This functional partnership establishes a crucial neurometabolic coupling that is the cornerstone of brain energy metabolism, enabling glia to sense neuronal energy needs and adjust the supply accordingly (Iadecola and Nedergaard, 2007; Mathiisen et al., 2010).

While both neurons and glia contribute toward brain energy metabolism, neuronal demands outweigh glia's by far (Attwell and Laughlin, 2001; Alle et al., 2009; Nortley and Attwell, 2017). The main energy expenditure of neurons is associated with re-establishing membrane potentials and enabling synaptic transmission (Alle et al., 2009; Harris et al., 2012; Rangaraju et al., 2014). Thereby the activity of ion pumps such as the $\mathrm{Na}^{+} / \mathrm{K}^{+}$ ATPase accounts for the majority of total energy spent in the entire brain (Attwell and Laughlin, 2001; Harris et al., 2012). This holds true not only for re-establishing membrane ion gradients after membrane depolarization, but also for maintaining baseline membrane potential by compensating spike-related ion shifts (Attwell and Laughlin, 2001). It has been estimated that maintaining baseline membrane potential is responsible for close to half of the total energy consumption in the white matter (Harris and Attwell, 2012) and about a quarter in cortical neurons (Lennie, 2003). Surprisingly, membrane depolarization by itself does not require large amounts of ATP, as action potentials (AP) can persist for some time without ATP utilization (Rangaraju et al., 2014). On the other hand, $\mathrm{Na}^{+} / \mathrm{K}^{+}$ATPase activity to 


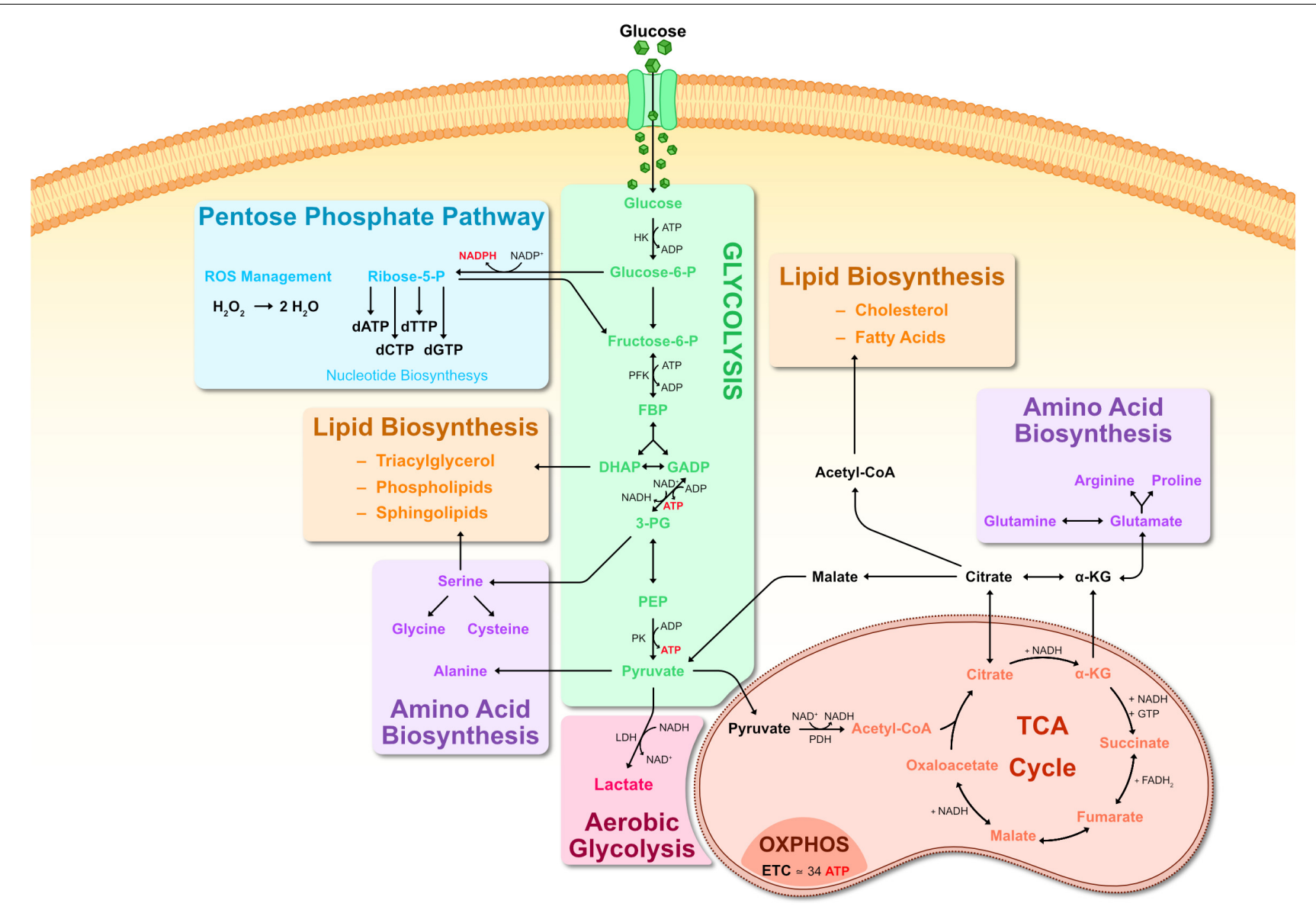

FIGURE 1 | Metabolic pathways of glucose metabolism. Representation of key steps in glucose metabolism. Upon entering the cell, glucose is first metabolized by HK into G6P, consuming ATP. G6P can branch off into the PPP pathway, producing R5P and generating large amounts of NADPH as a byproduct. R5P is a precursor for creating nucleic acids. NADPH generated by the PPP can be used to scavenge ROS via glutathione reduction, converting $\mathrm{H}_{2} \mathrm{O}_{2}$ into $\mathrm{H}_{2} \mathrm{O}$. $\mathrm{G} 6 \mathrm{P}$ is further metabolized into F6P and subsequently into FBP. FBP is metabolized into one molecule of DHAP and another of GADP. DHAP can branch off into lipid biosynthesis where it is a precursor for phospholipids and triacylglycerol. Otherwise, DHAP is transformed into GADP and both GADP molecules are metabolized into 3-PG, generating ATP in the process. 3-PG can be used for serine biosynthesis, which can then contribute toward synthesis of sphingolipids as well as glycine and cysteine. 3-PG goes on to be metabolized into PEP and then into pyruvate by PK activity. Pyruvate can: serve as substrate for alanine biosynthesis; be converted to lactate by LDH (known as anaerobic respiration or aerobic glycolysis); enter a mitochondrion where PDH metabolizes it into Acetyl-CoA. Acetyl-CoA then enters the TCA cycle, where it is metabolized in a series of sequential reactions resulting in citrate, $\alpha-K G$, succinate, fumarate, malate, oxaloacetate which cycles back to citrate. Some of these reactions generate byproducts such as $\mathrm{NADH}$ and $\mathrm{FADH}_{2}$. $\mathrm{NADH}$ and FADH 2 are then used for OXPHOS, generating ATP in the inner mitochondrial membrane matrix. Abbreviations: $\alpha$-KG, $\alpha$-ketoglutarate; 3-PG, 3-phosphoglycerate; ADP, adenosine diphosphate; ATP, adenosine triphosphate; CoA, coenzyme A; dATP, deoxyadenosine triphosphate; dCTP, deoxycytidine triphosphate; dGTP, deoxyguanosine triphosphate; DHAP, dihydroxyacetone phosphate; dTTP, deoxythymidine triphosphate; ETC, electron transport chain; F6P, Fructose-6-phosphate; FADH, flavin adenine dinucleotide, reduced; FBP, fructose 1,6-biphosphate; G6P, glucose-6-phosphate; GADP, glyceraldehyde-3-phosphate; GTP, guanosine triphosphate; HK, hexokinase; LDH, lactate dehydrogenase; NAD, nicotinamide adenine dinucleotide; NADH, nicotinamide adenine dinucleotide, reduced; NADP, nicotinamide adenine dinucleotide phosphate; NADPH, nicotinamide adenine dinucleotide phosphate, reduced; OXPHOS, oxidative phosphorylation; PEP, phosphoenolpyruvate; PDH, pyruvate dehydrogenase; PK, pyruvate kinase; PPP, pentose phosphate pathway; TCA, tricarboxylic acid.

restore the proper $\mathrm{Na}^{+}$and $\mathrm{K}^{+}$gradients after an $\mathrm{AP}$ consumes an immense amount of ATP (up to $8 \times 10^{\wedge} 8$ molecules) (Lennie, 2003; Kole et al., 2008; Hallermann et al., 2012; Howarth et al., 2012). Interestingly, in synaptic transmission $\mathrm{Na}^{+} / \mathrm{K}^{+}$ATPase activity represents only a small fraction of energy expenditure compared to the requirements of the synaptic vesicle cycle (Alle et al., 2009; Rangaraju et al., 2014; Yuan et al., 2018).

While neurons are primarily oxidative in their metabolism, relying on OXPHOS activity to generate ATP in an activity-dependent manner (Verstreken et al., 2005;
Ly and Verstreken, 2006; Lin et al., 2010; Hall et al., 2012), astrocytes are highly glycolytic (Herrero-Mendez et al., 2009; Bittner et al., 2011; Figure 2). Several factors determine astrocytes' metabolic profile: First, astrocytes have an increased expression and activity of Phosphofructokinase (PFK), thus promoting glycolysis (Herrero-Mendez et al., 2009; Bélanger et al., 2011; Zhang et al., 2014). Second, astrocytes express lactate dehydrogenase 5 (LDH5), an enzyme whose subunit composition favors catalysis of pyruvate into lactate (O'Brien et al., 2007; Zhang et al., 2014; Duka et al., 2017; Mason, 2017). Finally, 


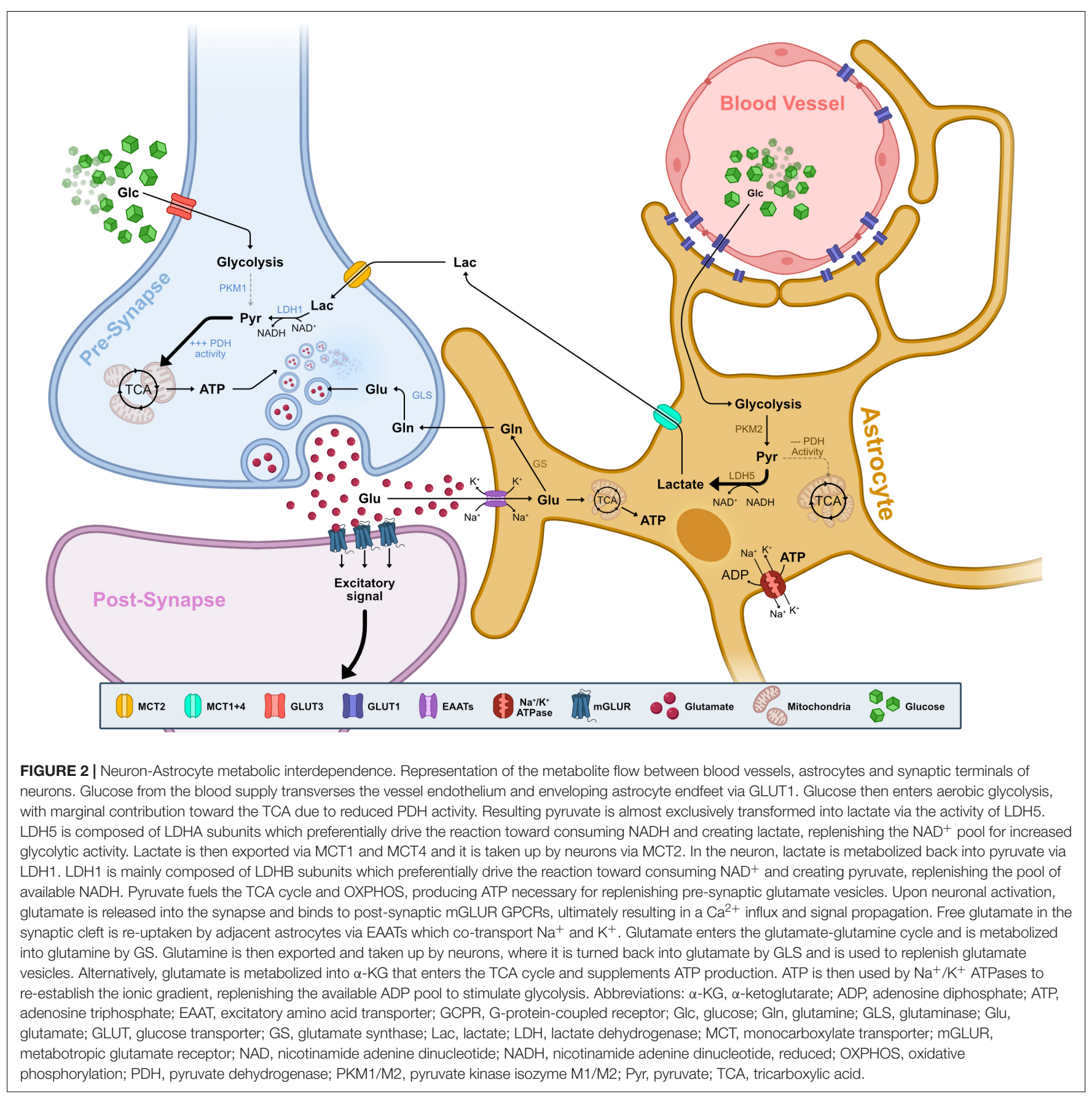

astrocytes have reduced pyruvate dehydrogenase (PDH) activity, which means that any increase from basal pyruvate levels diverts into LDH5-mediated conversion to lactate instead of entry into TCA cycle and OXPHOS activity (Halim et al., 2010; Hirrlinger and Dringen, 2010; Zhang et al., 2014; Figure 2).

Conversely, neuronal glycolysis levels are negatively impacted by decreased synthesis and activity of PFK (Herrero-Mendez et al., 2009), an inability of neuronal pyruvate kinase (PKM1) to regulate glycolytic flux and finally, by expressing LDH1, a LDH isozyme whose subunit composition kinetically favors catalysis of lactate to pyruvate (Ward and Thompson, 2012;
Zhang et al., 2014). The attenuation of glycolysis in neurons allows glucose to be diverted to the PPP in order to offset ROS production. Accordingly, upregulating glycolysis in neurons is met with increased oxidative stress and cell death (Gavillet et al., 2008; Herrero-Mendez et al., 2009; Bolaños et al., 2010).

The difference between neurons and astrocytes briefly outlined here is at the basis of their partnership and significantly shapes brain energy metabolism. This interdependence is formulated in the astrocyte-neuron lactate shuttle hypothesis (ANLS), one of the most widely accepted models describing brain energy metabolism at the cellular level 
(Pellerin and Magistretti, 1994; Pellerin et al., 1998; Allaman et al., 2011; Magistretti and Allaman, 2015). Underlying this model is the notion that even though glucose is the sole energy source of the brain, glucose-derived metabolites such as lactate can themselves serve as an alternate substrate for energy production.

The main postulate of the ANLS is that neuronal activation triggers an increase of astrocyte glucose uptake, which is then mainly converted into lactate and shuttled to neurons where it can be used as a substrate for oxidative energy metabolism (Pellerin and Magistretti, 1994; Magistretti and Allaman, 2015; Figure 2). In the ANLS seminal study, Pellerin and Magistretti demonstrated that glucose uptake and consequent lactate production in cultured astrocytes is stimulated by the excitatory neurotransmitter glutamate (Pellerin and Magistretti, 1994).

Glutamate released into the synaptic cleft is taken up by excitatory amino acid transporters (EAATs), which are present in glial lamellar processes sheeting the post- and pre-synaptic elements (Iadecola and Nedergaard, 2007). The extent of glutamate import serves as a neuronal activity measure and glutamate uptake by EAATs leads to a concomitant influx of $\mathrm{Na}^{+}$and efflux of $\mathrm{K}^{+}$. This triggers $\mathrm{Na}^{+} / \mathrm{K}^{+}$ATPase activity thereby decreasing local ATP concentration, which in turn promotes glycolysis in astrocytes (Barros et al., 2009; Herrero-Mendez et al., 2009; Chuquet et al., 2010; Bittner et al., 2011; Figure 2). Like neurons, astrocytes differentially express a pyruvate kinase isoform (PKM2) that, in contrast to the neuronal isoform, is capable of regulating glycolytic flux in response to increased energy needs (Zhang et al., 2014; Casson et al., 2016).

Astrocytic glycolysis feeds into lactate production which is released through dedicated monocarboxylate transporters (MCTs) (Pellerin et al., 2005). Neurons then take up extracellular lactate via neuron specific MCT where it is subsequently converted it into pyruvate by LDH1. Pyruvate then enters the TCA cycle, ultimately producing between 14 and 17 molecules of ATP through OXPHOS per molecule of lactate (Pellerin and Magistretti, 1994; Pellerin et al., 2005; Magistretti, 2006; Halestrap, 2013; Figure 2).

The large majority of the initial work behind the ANLS and in the years following its inception is based on in vitro cell models of neurons and astrocytes. Controversies concerning the ANLS mostly surrounded the question how readily these in vitro results are applicable to the situation in the intact brain (Tang, 2018; Dienel, 2019). However, as current research starts to focus on tissue culture and in vivo models, most of these questions are getting answered (Barros et al., 2009; van Hall et al., 2009; Chuquet et al., 2010; Wyss et al., 2011; Fünfschilling et al., 2012; Jakoby et al., 2014; Sotelo-Hitschfeld et al., 2015; Volkenhoff et al., 2015; Mongeon et al., 2016; Mächler et al., 2016; Clasadonte et al., 2017; Mazuel et al., 2017; Supplie et al., 2017; Zimmer et al., 2017; Ruminot et al., 2019). While the ANLS has been proposed to explain the metabolic landscape for the whole central nervous system (CNS), the canonical ANLS model cannot fully describe the observed metabolic interactions in the retina, despite being a part of the CNS. In the following section, we will consolidate and conceptually frame what is known about the retinal energy metabolism.

\section{RETINAL STRUCTURE AND FUNCTION}

In humans, vision is the primary sense and a large portion of the neocortex is dedicated to the processing of visual information. The first step in visual perception is light detection and preprocessing by the neural retina (Figure 3A). This stereotypically layered tissue has three nuclear layers (ONL, INL, GCL) and two synaptic layers (OPL and IPL) containing a total of five different neuronal and one glial cell type (Figure 3B). The ONL contains rod and cone photoreceptors, whereas cell bodies of bipolar, amacrine and horizontal cells, as well as Müller glia make up the INL. The third layer is the GCL, which contains ganglion cells as well as displaced amacrine cells. All of these cells contribute toward modulating and processing signals generated by photoreceptors in response to light (Dowling, 1970; Baden et al., 2020; Figure 3B).

Photoreceptors are the light-sensitive neurons, which are responsible for converting light into a biological signal in a process called phototransduction. Both rod and cone photoreceptors have an outer segment (OS) that contains membranous disks stacked with light-sensitive photopigments (Figure 3C). The photoreceptor outer segment is in direct contact with the retinal pigment epithelium (RPE), involved in numerous support functions and the apical part of the RPE is in contact with the choroidal vasculature (Bok, 1993; Figure 3B). This connection represents the photoreceptors' main source for essential nutrients, establishing a major metabolite supply-line for photoreceptors as part of the Blood-Retina Barrier (BRB) (Campbell and Humphries, 2012).

\section{RETINAL ENERGY METABOLISM}

The particularities of photoreceptor form and function are at the root of their high energy requirements (Wong-Riley, 2010; Country, 2017). Otto Warburg was the first to report on the levels of oxygen consumption of the retina, which was the highest of any tissue of the body that he tested, comparable only to rapidly proliferating cancer cells (Warburg et al., 1924; Warburg, 1956). Warburg first described the eponymous Warburg effect as a metabolic switch to glycolysis in the presence of oxygen (aerobic glycolysis) displayed by tumor cells. In addition to cancer cells, the Warburg effect is also found in the retina (Ng et al., 2015; DeBerardinis and Chandel, 2020). This is surprising, since in contrast to cancer cells, retinal neurons are postmitotic.

Apart from the high catabolic needs of photoreceptors, they display extraordinary anabolic needs. This is due to the curious phenomenon of outer segment renewal, where photoreceptors are continuously shedding the outer most part of the outer segments, amounting to about 10\% per day (Young, 1967, 1971). Consequently, new disks have to be continuously added at the base of the outer segment, requiring a steady supply of a large number of complex macromolecules such as lipids and proteins 

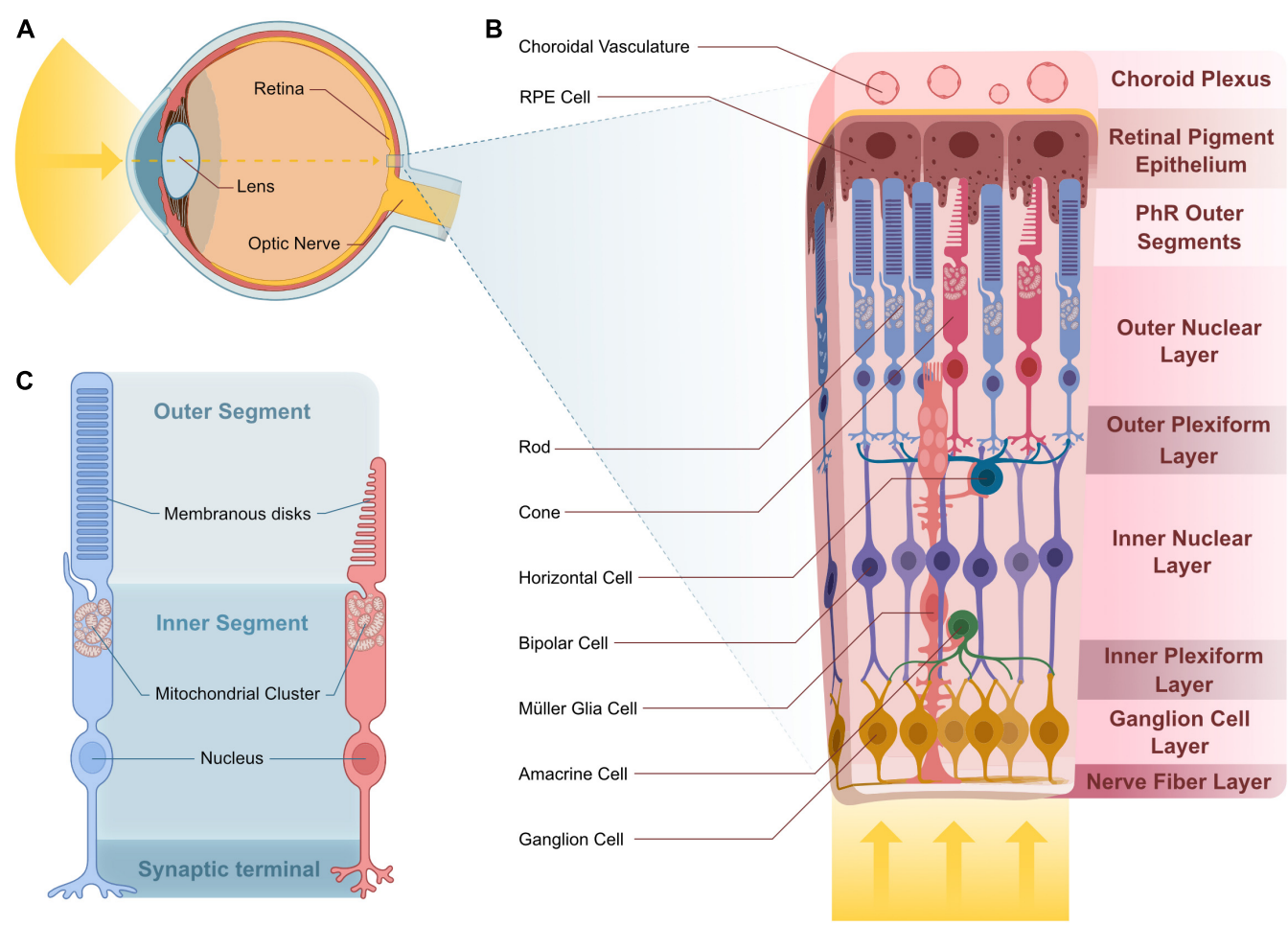

FIGURE 3 | Vertebrate retina structure and cellular composition. (A) Sagittal representation of the human eye. Light enters the eye through the lens and is projected onto the retina. (B) Stereotypical multilayered structure of the canonical vertebrate retina. Light transverses the entire retina until it reaches the photosensible PhRs at the distal ONL, in contact with RPE projections that are adjacent to the choroid plexus where choroidal vasculature supplies nutrients to the outer retina. In the OPL, $\mathrm{PhR}$ terminals contact projections from $\mathrm{HC}$ and $\mathrm{BC}$, whose cell body reside in the INL. Projections from BC contact those from the GC and AC in the IPL. GC cell bodies reside in the GCL and their projections bundle in the NFL to create the optic nerve. MGCs span all of these layers and support the cells they contact. (C) Rod and cone PhRs cell structure. PhRs have an inner and outer segment. PhR OSs house membranous disks that house the photosensitive pigment responsible for light detection. The IS consists of the rest of the cell body and houses a mitochondrial cluster at the base of the OS. Abbreviations: AC, amacrine cell; BC, bipolar cell; GC, ganglion cell; HC, horizontal cell; INL, inner nuclear layer; IPL, inner plexiform layer; IS, inner segment; MGC, Müller glia cell; NFL, nerve fiber layer; ONL, outer nuclear layer; OPL, outer plexiform layer; OS, outer segment; PhR, photoreceptor; RPE, retinal pigment epithelium.

to maintain proper function and OS length (Wang et al., 2011, 2016; Park et al., 2018; Léveillard et al., 2019).

The retina, much like the rest of the CNS, preferentially uses glucose as its main energy substrate. Glucose supply of the retina is mainly mediated by glucose transporters ( $\mathrm{Hsu}$ and Molday, 1991; Gospe et al., 2010), which are expressed in vasculature, RPE cells, PhRs and in MGCs (Watanabe et al., 1996; WongRiley, 2010; Veys et al., 2020; Figure 4). GLUTs are facilitative transporters and as such glucose follows a concentration gradient (Cheeseman and Long, 2015; Holman, 2020). Glucose from the blood supply leaves the choroidal endothelium and diffuses through RPE cells into the photoreceptor layer where photoreceptors take it up and readily metabolize it. This facilitated diffusion is thought to be mediated by GLUT1 (WongRiley, 2010; Narayan et al., 2017).

Like other neurons, the largest fraction of ATP expenditure within photoreceptors is active ion transport and membrane potential repolarization. ATP consumption in photoreceptors is activity dependent and substantially different in darkness and in light (Okawa et al., 2008; Hurley et al., 2015). The glutamate dependent dark current is energetically expensive and requires a large amount of ATP to sustain. In mice, a single rod photoreceptor consumes about four times more ATP in darkness than in light (approximately $10^{8}$ ATP $\mathrm{s}^{-1}$ ) (Okawa et al., 2008). Excess $\mathrm{Ca}^{2+}$ and $\mathrm{Na}^{+}$is actively transported out of the cell via ATPase pumps and carries a significant energy cost. The combined cost for this efflux is almost $9 \times 10^{7} \mathrm{ATP} \mathrm{s}^{-1}$. Interestingly, energy profiles for rods and cones differ quite substantially. In general, cones have higher energy needs than rods, probably due to differences in energy requirement of the dark current, phototransduction, and synaptic release (Okawa et al., 2008; Ingram et al., 2020).

As in most cells, a pivotal role of glucose in the retina is enabling ATP production to meet the cell's energy demands (Ames et al., 1992; Ng et al., 2015; Narayan et al., 2017). Both glycolysis and OXPHOS generate ATP, although OXPHOS yields are far higher. It is by now well established that photoreceptors produce lactate in the presence of oxygen (Hurley et al., 2015; Kanow et al., 2017). As previously mentioned, aerobic glycolysis is also frequently referred to as the Warburg effect, which is a frequent trait of fast dividing cancer cells and neural stem cells (Namba et al., 2020). Why would energy-demanding cells like photoreceptors opt out of using OXPHOS, the most energy profitable pathway? At least a partial explanation may 


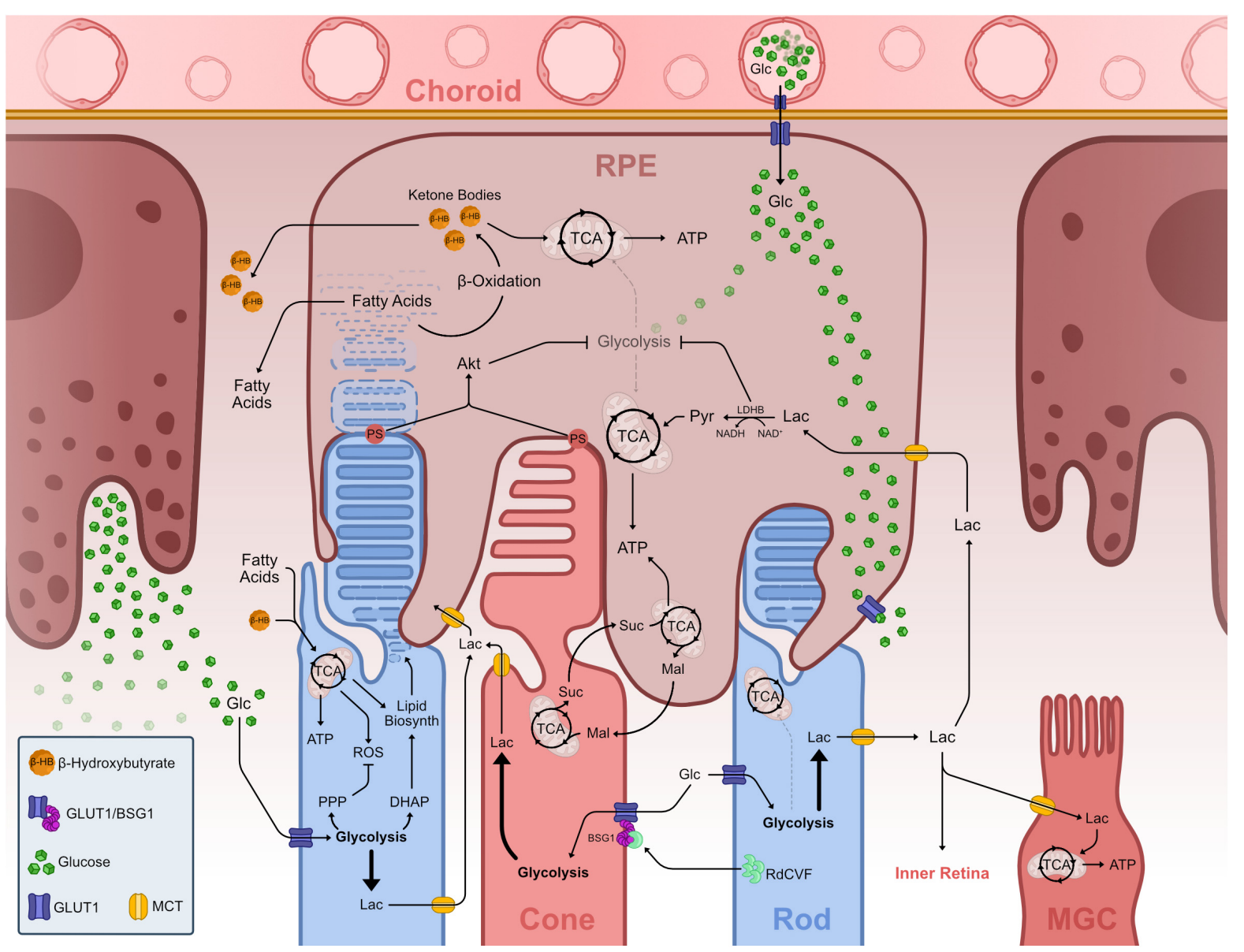

FIGURE 4 | Metabolic landscape of the vertebrate outer retina. Representation of known metabolic interactions of cells within the outer retina. Glucose from the choroidal blood supply transverses the endothelium and enters the RPE via GLUT1. In the RPE, glucose flows through the cell un-metabolized, thus reaching the basal membrane adjacent to PhR OS. Glucose leaves the RPE via GLUT1 into the PhR layer. PhRs take up glucose via GLUT1 and use it to fuel aerobic glycolysis. Aerobic glycolysis in PhRs fuels the PPP, creating nucleic acids and offsetting ROS production from mitochondrial activity, and creates DHAP which is used to synthesize phospholipids necessary to create OS disks thus driving OS growth and PhR function. Lactate from aerobic glycolysis is transported from PhRs to the extracellular space where it is taken up by other cells such as MGC and RPE, fueling TCA cycle and OXPHOS in these cells. RPE cells take up lactate and transform into pyruvate, which enters the TCA. This LDHB activity depletes available NAD+, effectively inhibiting glycolysis, allowing glucose to reach GLUTs in the basal RPE membrane. Extending OS tips externalize PS and contact with RPE projections and subsequent phagocytosis leads to Akt activation, which also downregulates glycolysis in these cells. PhR OS phagocytosis in the RPE leads to an increase in available fatty acids from breaking down the membranous lipid disks. These fatty acids undergo $\beta$-oxidation creating ketone bodies in the form of $\beta$-hydroxybutyrate, which can be further metabolized and used in the TCA cycle, supplementing RPE mitochondrial activity. Both fatty acids and ketone bodies are transported to the PhR layer where they are taken up by these and used to supplement TCA cycle and OXPHOS. A reciprocal malate-succinate shuttle between PhRs and RPE cells exists that exchanges reducing power between these cells. In PhR, reverse SDH activity, due to hypoxia-induced decrease of COXIV, produces succinate that is exported to RPE cells, where it is oxidized in the TCA cycle and generates a surplus of malate. Malate is then exported to the PhR layer, enters the TCA cycle and fuels succinate production. Rods effectively promote cone survival and function by secreting RdCVF, which binds and makes a complex with BSG1 and GLUT1 in the cone membrane, promoting glucose supply to these cells. Abbreviations: ADP, adenosine diphosphate; Akt, protein kinase B; ATP, adenosine triphosphate; BSG1, basigin 1; COXIV, cytochrome c oxidase subunit 4; DHAP, dihydroxyacetone phosphate; Glc, glucose; GLUT, glucose transporter; Lac, lactate; LDH, lactate dehydrogenase; Mal, malate; MCT, monocarboxylate transporter; MGC, Müller glia cell; NAD, nicotinamide adenine dinucleotide; NADH, nicotinamide adenine dinucleotide, reduced; OS, outer segment; OXPHOS, oxidative phosphorylation; PDH, pyruvate dehydrogenase; PhR, photoreceptor; PPP, pentose phosphate pathway; PS, phosphatidylserine; Pyr, pyruvate; RdCVF, rod-derived cone viability factor; ROS, reactive oxygen species; RPE, retinal pigment epithelium; SDH, succinate dehydrogenase; Suc, succinate; TCA, tricarboxylic acid.

lie in the strong need for anabolic intermediates, provided by glycolysis (Casson et al., 2016; Narayan et al., 2017). Although photoreceptors do not proliferate, they nevertheless have an immense anabolic needs due to the constant renewal of their outer segments (Young, 1967, 1971; Ng et al., 2015).

Although the fascination with retinal energy metabolism has an almost century-long history starting with Warburg, more recent work has now laid the foundation for a more integrative view of retinal energy metabolism. The focus is no longer solely on photoreceptors but on the metabolic network of neighboring cells in the outer retina forming a metabolic landscape (Figure 4). The first consideration is how glucose enters the retina. Glucose is provided by blood circulation and has to pass the blood retina barrier (BRB). It is shuttled through the choroidal endothelium 
into adjacent RPE cells, before reaching the outer retina. RPE shuttles most glucose via GLUT1 to the photoreceptor layer, while relying on lactate provided by photoreceptors for its own metabolism (Badr et al., 2000; Gospe et al., 2010; Kanow et al., 2017; Figure 4). RPE cells minimize glucose consumption and maximize glucose efflux by using photoreceptor-derived lactate as their metabolic substrate. Concomitantly, lactate is a negative feedback regulator of glycolysis that dampens RPE glucose consumption (Figure 4; Kanow et al., 2017). This intimate metabolic co-dependence is not restricted to catabolism, but extends to anabolism. RPE cells export malate for photoreceptors to replenish succinate that is exported back to RPE cells. This enables RPE cells to generate reductive power in the form of $\mathrm{NADPH}$ regenerating malate in the process, thereby closing the cycle (Bisbach et al., 2020; Figure 4). Malate can also be converted to pyruvate, yielding additional NADPH in the process (Adler et al., 2014; Figures 1, 4). RPE cells are also known to rely on $\beta$-oxidation of fatty acids, resulting from phagocytosed disks of shed photoreceptor OS, to generate energy. The resulting ketone bodies supplement the TCA cycle, providing ATP and metabolic intermediates, and therefore compensate for the downregulation of glycolysis (Figure 4; Adijanto et al., 2014; Reyes-Reveles et al., 2017; Fu et al., 2020). Moreover, the RPE has been shown to supply photoreceptors with fatty acids and ketone bodies, which can be metabolized in the mitochondria and supplement TCA cycle activity and ATP production (Figure 4; Adijanto et al., 2014; Léveillard and Sahel, 2017; Fu et al., 2020). Another hallmark of the interdependence between photoreceptor outer segments and the RPE is the recent finding that contacts between phosphatidylserine on outer segment tips and RPE receptors activates the intracellular signaling Akt pathway. This effectively links phagocytosis with glucose transport to photoreceptors for new outer segment synthesis (Wang et al., 2019; Figure 4).

Taken together, these studies establish a close metabolic interaction between the RPE and the outer retina regarding the exchange of not only glucose and lactate, but also metabolites involved in anabolism. The involvement of aerobic glycolysis in biogenesis and maintenance of outer segment has been established also in rod photoreceptors, by interfering with key enzymes mediating aerobic glycolysis (Chinchore et al., 2017; Petit et al., 2018).

In the metabolic landscape there are not only reciprocal interactions between the RPE and photoreceptors, but also between rods and cones. The interdependence of photoreceptors has been known for long by the puzzling bystander effect where genetically compromised rods lead to the demise of uncompromised cones (Ripps, 2002). This effect is proposed to be due to the absence of the rod-derived cone viability factor (RdCVF) resulting from rod degeneration. The splice variant of the Nxnl1genes encodes RdCVF, which binds to Basigin-1 (BSG1) expressed on the cell surface of cones. BSG1 forms a complex with GLUT1, stimulating glucose uptake upon RdCVF binding and consequently aerobic glycolysis (Léveillard et al., 2004; AïtAli et al., 2015; Figure 4). The absence of this interaction metabolically starves cones.

In the proposed metabolic landscape scenario, photoreceptors are set as energy relay nodes using glucose to meet their anabolic and catabolic needs. At the same time, they provide surrounding cells with metabolic intermediates (Figure 4). This is a fascinating variation on the ANLS in other parts of the CNS. We saw how the ANLS depicts a metabolic exchange between astrocytes and neurons where astrocytic lactate from aerobic glycolysis helps fuel neuronal activity. Within the retinal metabolic landscape, photoreceptors take on a similar role to astrocytes in the rest of the CNS by supplying lactate to surrounding cells. Moreover, both astrocytes in the CNS and photoreceptors in the retina are the first neural cells to contact glucose in their respective system. This curious "role-reversal" may very well be an adaption to the unique microenvironment of the retina and the extraordinary anabolic needs of postmitotic photoreceptors. The large demand of glycolytic intermediates for anabolism makes photoreceptors a glucose sink that surrounding cells have adapted to by using by-products to support their own metabolic needs (Kanow et al., 2017).

This model suggests an intricate and interdependent cell environment, yet there are some interesting aspects that await further clarification. Among them is the metabolic distinction between rod and cone photoreceptors and use-dependent metabolic requirements. Another curious outstanding question is how photoreceptors cope with the low ATP-yielding aerobic glycolysis to support oxidative metabolism alone. Moreover, if this is the case then why do photoreceptors have large, tightly packed mitochondrial clusters adjacent to the OS (Giarmarco et al., 2020)? While the current retinal metabolic landscape specifies many interactions within the retina from a bioenergetics point-of-view, it is far from complete and many of the proposed interactions would benefit from additional robustness and independent confirmation. Furthermore, almost all of the supporting data is from murine models that rely mainly on scotopic (nocturnal) vision and whose retina is composed of only about $2 \%$ cones. The rest of the data is from in vitro cell modeling of the retina. While both these approaches have proven important, there is a pressing need for further in vivo data avoiding more artificial in vitro methods. This is particularly true for information on cone photoreceptors, which mediate high-acuity color vision that humans rely on.

\section{RETINAL METABOLISM IN DISEASE}

We have summarized recent evidence that glucose metabolism is the key to maintaining brain and retina homeostasis. Given the strong dependence of neural cells on glucose metabolism, it comes as no surprise that metabolism is frequently affected in neural diseases, be it the cause or the results of metabolic perturbations. The retina is no exception to this and several retinal diseases have been linked to metabolism.

A case in point are the pathomechanisms of outer retinal dystrophies, such as of age-related macular degeneration (AMD) and retinitis pigmentosa (RP). In this group of diseases, there is a growing realization that metabolic alterations may lie at the heart of their pathophysiology (Narayan et al., 2016; Campochiaro and Mir, 2018; Léveillard et al., 2019). 
Age-related macular degeneration is a retinal disease that is the leading cause of blindness in seniors worldwide (Mitchell et al., 2018). While the mechanisms behind AMD have yet to be uncovered, several recent studies point to oxidative damage (Ferrington et al., 2017; Hwang et al., 2019; Terluk et al., 2019) and mitochondrial dysfunction as key steps in AMD pathophysiology (Karunadharma et al., 2010; Terluk et al., 2015; Ebeling et al., 2020). The concept of a retinal metabolic landscape can help to link accumulated mitochondrial DNA damage in the RPE to macular degeneration. RPE cells with compromised mitochondrial function may shift toward increased glucose consumption and thereby compromise photoreceptor access to glucose. Conversely, changes in photoreceptor energy supply will have repercussions on the RPE.

Retinitis pigmentosa (RP) is the disease most tightly linked to changes in the retinal metabolic landscape. The retinal metabolic landscape brought forward here was strongly driven by studies related to RP (Aït-Ali et al., 2015; Wang et al., 2016, 2019; Petit et al., 2018). RP describes a group of rare diseases where mostly rod-specific mutations cause rod, and subsequently cone, degeneration (Hartong et al., 2006). The reason behind this bystander effect can be attributed to the lack of cone viability factor (RdCVF), which ultimately stimulates cone glucose uptake (Aït-Ali et al., 2015).

As the intricacies of the retinal metabolic landscape start to unravel, we begin to see how energy homeostasis is crucial

\section{REFERENCES}

Adijanto, J., Du, J., Moffat, C., Seifert, E. L., Hurle, J. B., and Philp, N. J. (2014). The retinal pigment epithelium utilizes fatty acids for ketogenesis. J. Biol. Chem. 289, 20570-20582. doi: 10.1074/jbc.M114.565457

Adler, L., Chen, C., and Koutalos, Y. (2014). Mitochondria contribute to NADPH generation in mouse rod photoreceptors. J. Biol. Chem. 289, 1519-1528. doi: 10.1074/jbc.M113.511295

Aït-Ali, N., Fridlich, R., Millet-Puel, G., Clérin, E., Delalande, F., Jaillard, C., et al. (2015). Rod-derived cone viability factor promotes cone survival by stimulating aerobic glycolysis. Cell 161, 817-832. doi: 10.1016/j.cell.2015. 03.023

Allaman, I., Bélanger, M., and Magistretti, P. J. (2011). Astrocyte-neuron metabolic relationships: for better and for worse. Trends Neurosci. 34, 76-87. doi: 10.1016/ j.tins.2010.12.001

Alle, H., Roth, A., and Geiger, J. R. P. (2009). Energy-efficient action potentials in hippocampal mossy fibers. Science 325, 1405-1408. doi: 10.1126/science. 1174331

Ames, A. (2000). CNS energy metabolism as related to function. Brain Res. Rev. 34, 42-68. doi: 10.1016/S0165-0173(00)00038-2

Ames, A., Li, Y. Y., Heher, E. C., and Kimble, C. R. (1992). Energy metabolism of rabbit retina as related to function: high cost of $\mathrm{Na}+$ transport. J. Neurosci. 12, 840-853. doi: 10.1523/JNEUROSCI.12-03-00840.1992

Ashrafi, G., and Ryan, T. A. (2017). Glucose metabolism in nerve terminals. Curr. Opin. Neurobiol. 45, 156-161. doi: 10.1016/j.conb.2017.03.007

Attwell, D., Buchan, A. M., Charpak, S., Lauritzen, M., MacVicar, B. A., and Newman, E. A. (2010). Glial and neuronal control of brain blood flow. Nature 468, 232-243. doi: 10.1038/nature09613

Attwell, D., and Laughlin, S. B. (2001). An energy budget for signaling in the grey matter of the brain. J. Cereb. Blood Flow Metab. 21, 1133-1145. doi: 10.1097/ 00004647-200110000-00001

Baden, T., Euler, T., and Berens, P. (2020). Understanding the retinal basis of vision across species. Nat. Rev. Neurosci. 21, 5-20. doi: 10.1038/s41583-019-0242-1 to maintain a proper functioning system. Upsetting metabolic interactions can have significant consequences leading to specific disease phenotypes. Furthering our knowledge of this fascinating retinal landscape on any level is bound to have a large impact on what is already known and has yet to be discovered about these diseases, as well as possibly contributing to the development of therapeutic strategies to combat them.

\section{AUTHOR CONTRIBUTIONS}

FV and SN drafted, wrote, and edited the manuscript. FV designed and drew the figures. Both authors contributed to the article and approved the submitted version.

\section{FUNDING}

This work was supported by the Swiss National Science Foundation (31003A_173083).

\section{ACKNOWLEDGMENTS}

We would like to thank Drs. Sara Haddad and Matthias Gesemann for critical comments on the manuscript. Some icons in the figures are based on icons purchased from biorender.com.

Badr, G. A., Tang, J., Ismail-Beigi, F., and Kern, T. S. (2000). Diabetes downregulates GLUT1 expression in the retina and its microvessels but not in the cerebral cortex or its microvessels. Diabetes 49, 1016-1021. doi: 10.2337/ diabetes.49.6.1016

Barros, L. F., Brown, A., and Swanson, R. A. (2018). Glia in brain energy metabolism: a perspective. Glia 66, 1134-1137. doi: 10.1002/glia.23316

Barros, L. F., Courjaret, R., Jakoby, P., Loaiza, A., Lohr, C., and Deitmer, J. W. (2009). Preferential transport and metabolism of glucose in Bergmann glia over Purkinje cells: a multiphoton study of cerebellar slices. Glia 57, 962-970. doi: 10.1002/glia.20820

Bélanger, M., Allaman, I., and Magistretti, P. J. (2011). Brain energy metabolism: focus on astrocyte-neuron metabolic cooperation. Cell Metab. 14, 724-738. doi: 10.1016/j.cmet.2011.08.016

Bisbach, C. M., Hass, D. T., Robbings, B. M., Rountree, A. M., Sadilek, M., Sweet, I. R., et al. (2020). Succinate can shuttle reducing power from the hypoxic retina to the O2-Rich pigment epithelium. Cell Rep. 31:107606. doi: 10.1016/j.celrep. 2020.107606

Bittner, C. X., Valdebenito, R., Ruminot, I., Loaiza, A., Larenas, V., SoteloHitschfeld, T., et al. (2011). Fast and reversible stimulation of astrocytic glycolysis by $\mathrm{K}+$ and a delayed and persistent effect of glutamate. J. Neurosci. 31, 4709-4713. doi: 10.1523/JNEUROSCI.5311-10.2011

Bok, D. (1993). The retinal pigment epithelium: a versatile partner in vision. J. Cell Sci. Suppl. 17, 189-195. doi: 10.1242/jcs.1993.supplement_17.27

Bolaños, J. P., Almeida, A., and Moncada, S. (2010). Glycolysis: a bioenergetic or a survival pathway? Trends Biochem. Sci. 35, 145-149. doi: 10.1016/j.tibs.2009.10. 006

Campbell, M., and Humphries, P. (2012). The blood-retina barrier: tight junctions and barrier modulation. Adv. Exp. Med. Biol. 763, 70-84.

Campochiaro, P. A., and Mir, T. A. (2018). The mechanism of cone cell death in Retinitis Pigmentosa. Prog. Retin. Eye Res. 62, 24-37. doi: 10.1016/j.preteyeres. 2017.08.004

Casson, R. J., Wood, J. P. M., Han, G., Kittipassorn, T., Peet, D. J., and Chidlow, G. (2016). M-Type pyruvate kinase isoforms and lactate dehydrogenase A in 
the mammalian retina: metabolic implications. Invest. Opthalmol. Vis. Sci. 57, 66-80. doi: 10.1167/iovs.15-17962

Cheeseman, C., and Long, W. (2015). Structure of, and functional insight into the GLUT family of membrane transporters. Cell Health Cytoskeleton 7, 167-183. doi: 10.2147/CHC.S60484

Chinchore, Y., Begaj, T., Wu, D., Drokhlyansky, E., and Cepko, C. L. (2017). Glycolytic reliance promotes anabolism in photoreceptors. Elife 6:e25946. doi: 10.7554/eLife.25946

Chuquet, J., Quilichini, P., Nimchinsky, E. A., and Buzsáki, G. (2010). Predominant enhancement of glucose uptake in astrocytes versus neurons during activation of the somatosensory cortex. J. Neurosci. 30, 15298-15303. doi: 10.1523/ JNEUROSCI.0762-10.2010

Clasadonte, J., Scemes, E., Wang, Z., Boison, D., and Haydon, P. G. (2017). Connexin 43-mediated astroglial metabolic networks contribute to the regulation of the sleep-wake cycle. Neuron 95, 1365-1380.e5. doi: 10.1016/j. neuron.2017.08.022

Country, M. W. (2017). Retinal metabolism: a comparative look at energetics in the retina. Brain Res. 1672, 50-57. doi: 10.1016/j.brainres.2017. 07.025

DeBerardinis, R. J., and Chandel, N. S. (2020). We need to talk about the Warburg effect. Nat. Metab. 2, 127-129. doi: 10.1038/s42255-020-0172-2

Dienel, G. A. (2019). Brain glucose metabolism: integration of energetics with function. Physiol. Rev. 99, 949-1045. doi: 10.1152/physrev.00062.2017

Dowling, J. E. (1970). Organization of vertebrate retinas. Invest. Ophthalmol. 9, 655-680.

Duka, T., Collins, Z., Anderson, S. M., Raghanti, M. A., Ely, J. J., Hof, P. R., et al. (2017). Divergent lactate dehydrogenase isoenzyme profile in cellular compartments of primate forebrain structures. Mol. Cell. Neurosci. 82, 137-142. doi: 10.1016/j.mcn.2017.04.007

Ebeling, M. C., Polanco, J. R., Qu, J., Tu, C., Montezuma, S. R., and Ferrington, D. A. (2020). Improving retinal mitochondrial function as a treatment for agerelated macular degeneration. Redox Biol. 34:101552. doi: 10.1016/j.redox.2020. 101552

Ferrington, D. A., Ebeling, M. C., Kapphahn, R. J., Terluk, M. R., Fisher, C. R., Polanco, J. R., et al. (2017). Altered bioenergetics and enhanced resistance to oxidative stress in human retinal pigment epithelial cells from donors with agerelated macular degeneration. Redox Biol. 13, 255-265. doi: 10.1016/j.redox. 2017.05.015

Fu, Z., Kern, T. S., Hellström, A., and Smith, L. E. (2021). Fatty acid oxidation and photoreceptor metabolic needs. J. Lipid Res. 62:100035. doi: 10.1194/jlr. TR120000618

Fünfschilling, U., Supplie, L. M., Mahad, D., Boretius, S., Saab, A. S., Edgar, J., et al. (2012). Glycolytic oligodendrocytes maintain myelin and long-term axonal integrity. Nature 485, 517-521. doi: 10.1038/nature11007

Gavillet, M., Allaman, I., and Magistretti, P. J. (2008). Modulation of astrocytic metabolic phenotype by proinflammatory cytokines. Glia 56, 975-989. doi: 10.1002/glia.20671

Giarmarco, M. M., Brock, D. C., Robbings, B. M., Cleghorn, W. M., Tsantilas, K. A., Kuch, K. C., et al. (2020). Daily mitochondrial dynamics in cone photoreceptors. Proc. Natl. Acad. Sci. U.S.A. 117, 28816-28827. doi: 10.1073/ pnas. 2007827117

Gordon, G. R. J., Choi, H. B., Rungta, R. L., Ellis-Davies, G. C. R., and MacVicar, B. A. (2008). Brain metabolism dictates the polarity of astrocyte control over arterioles. Nature 456, 745-749. doi: 10.1038/nature07525

Gospe, S. M., Baker, S. A., and Arshavsky, V. Y. (2010). Facilitative glucose transporter Glut1 is actively excluded from rod outer segments. J. Cell Sci. 123, 3639-3644. doi: 10.1242/jcs.072389

Halestrap, A. P. (2013). The SLC16 gene family - structure, role and regulation in health and disease. Mol. Aspects Med. 34, 337-349. doi: 10.1016/j.mam.2012.05. 003

Halim, N. D., Mcfate, T., Mohyeldin, A., Okagaki, P., Korotchkina, L. G., Patel, M. S., et al. (2010). Phosphorylation status of pyruvate dehydrogenase distinguishes metabolic phenotypes of cultured rat brain astrocytes and neurons. Glia 58, 1168-1176. doi: 10.1002/glia.20996

Hall, C. N., Klein-Flügge, M. C., Howarth, C., and Attwell, D. (2012). Oxidative phosphorylation, not glycolysis, powers presynaptic and postsynaptic mechanisms underlying brain information processing. J. Neurosci. 32, 89408951. doi: 10.1523/JNEUROSCI.0026-12.2012
Hallermann, S., Kock, C. P. J., de Stuart, G. J., and Kole, M. H. P. (2012). State and location dependence of action potential metabolic cost in cortical pyramidal neurons. Nat. Neurosci. 15, 1007-1014. doi: 10.1038/nn.3132

Harris, J. J., and Attwell, D. (2012). The energetics of CNS white matter. J. Neurosci. 32, 356-371. doi: 10.1523/JNEUROSCI.3430-11.2012

Harris, J. J., Jolivet, R., and Attwell, D. (2012). Synaptic energy use and supply. Neuron 75, 762-777. doi: 10.1016/j.neuron.2012.08.019

Hartong, D. T., Berson, E. L., and Dryja, T. P. (2006). Retinitis pigmentosa. Lancet 368, 1795-1809. doi: 10.1016/S0140-6736(06)69740-7

Herrero-Mendez, A., Almeida, A., Fernández, E., Maestre, C., Moncada, S., and Bolaños, J. P. (2009). The bioenergetic and antioxidant status of neurons is controlled by continuous degradation of a key glycolytic enzyme by APC/CCdh1. Nat. Cell Biol. 11, 747-752. doi: 10.1038/ncb1881

Hirrlinger, J., and Dringen, R. (2010). The cytosolic redox state of astrocytes: maintenance, regulation and functional implications for metabolite trafficking. Brain Res. Rev. 63, 177-188. doi: 10.1016/j.brainresrev.2009.10.003

Holman, G. D. (2020). Structure, function and regulation of mammalian glucose transporters of the SLC2 family. Pflugers Arch. 472, 1155-1175. doi: 10.1007/ s00424-020-02411-3

Howarth, C., Gleeson, P., and Attwell, D. (2012). Updated energy budgets for neural computation in the neocortex and cerebellum. J. Cereb. Blood Flow Metab. 32, 1222-1232. doi: 10.1038/jcbfm.2012.35

Hsu, S. C., and Molday, R. S. (1991). Glycolytic enzymes and a GLUT-1 glucose transporter in the outer segments of rod and cone photoreceptor cells. J. Biol. Chem. 266, 21745-21752.

Hurley, J. B., Lindsay, K. J., and Du, J. (2015). Glucose, lactate, and shuttling of metabolites in vertebrate retinas. J. Neurosci. Res. 93, 1079-1092. doi: 10.1002/ jnr. 23583

Hwang, N., Kwon, M.-Y., Woo, J. M., and Chung, S. W. (2019). Oxidative stress-induced pentraxin 3 expression human retinal pigment epithelial cells is involved in the pathogenesis of age-related macular degeneration. Int. J. Mol. Sci. 20:6028. doi: 10.3390/ijms20236028

Iadecola, C., and Nedergaard, M. (2007). Glial regulation of the cerebral microvasculature. Nat. Neurosci. 10, 1369-1376. doi: 10.1038/nn2003

Ingram, N. T., Fain, G. L., and Sampath, A. P. (2020). Elevated energy requirement of cone photoreceptors. Proc. Natl. Acad. Sci. U.S.A. 117, 19599-19603. doi: 10.1073/pnas.2001776117

Jakoby, P., Schmidt, E., Ruminot, I., Gutiérrez, R., Barros, L. F., and Deitmer, J. W. (2014). Higher transport and metabolism of glucose in astrocytes compared with neurons: a multiphoton study of hippocampal and cerebellar tissue slices. Cereb. Cortex 24, 222-231. doi: 10.1093/cercor/bhs309

Jha, M. K., and Morrison, B. M. (2018). Glia-neuron energy metabolism in health and diseases: new insights into the role of nervous system metabolic transporters. Exp. Neurol. 309, 23-31. doi: 10.1016/j.expneurol.2018.07.009

Kanow, M. A., Giarmarco, M. M., Jankowski, C. Sr., Tsantilas, K., Engel, A. L., Du, J., et al. (2017). Biochemical adaptations of the retina and retinal pigment epithelium support a metabolic ecosystem in the vertebrate eye. Elife 6:e28899. doi: 10.7554/eLife.28899

Karunadharma, P. P., Nordgaard, C. L., Olsen, T. W., and Ferrington, D. A. (2010). Mitochondrial DNA damage as a potential mechanism for age-related macular degeneration. Invest. Opthalmol. Vis. Sci. 51, 5470-5479. doi: 10.1167/iovs.105429

Kole, M. H. P., Ilschner, S. U., Kampa, B. M., Williams, S. R., Ruben, P. C., and Stuart, G. J. (2008). Action potential generation requires a high sodium channel density in the axon initial segment. Nat. Neurosci. 11, 178-186. doi: $10.1038 / \mathrm{nn} 2040$

Lennie, P. (2003). The cost of cortical computation. Curr. Biol. 13, 493-497. doi: 10.1016/S0960-9822(03)00135-0

Léveillard, T., Mohand-Saïd, S., Lorentz, O., Hicks, D., Fintz, A.-C., Clérin, E., et al. (2004). Identification and characterization of rod-derived cone viability factor. Nat. Genet. 36, 755-759. doi: 10.1038/ng1386

Léveillard, T., Philp, N. J., and Sennlaub, F. (2019). Is retinal metabolic dysfunction at the center of the pathogenesis of age-related macular degeneration? Int. J. Mol. Sci. 20:762. doi: 10.3390/ijms20030762

Léveillard, T., and Sahel, J.-A. (2017). Metabolic and redox signaling in the retina. Cell. Mol. Life Sci. 74, 3649-3665. doi: 10.1007/s00018-016-2318-7

Lin, A.-L., Fox, P. T., Hardies, J., Duong, T. Q., and Gao, J.-H. (2010). Nonlinear coupling between cerebral blood flow, oxygen consumption, and 
ATP production in human visual cortex. Proc. Natl. Acad. Sci. U.S.A. 107, 8446-8451. doi: 10.1073/pnas.0909711107

Lunt, S. Y., and Vander Heiden, M. G. (2011). Aerobic glycolysis: meeting the metabolic requirements of cell proliferation. Annu. Rev. Cell Dev. Biol. 27, 441-464. doi: 10.1146/annurev-cellbio-092910-154237

Ly, C. V., and Verstreken, P. (2006). Mitochondria at the synapse. Neuroscientist 12, 291-299. doi: $10.1177 / 1073858406287661$

Mächler, P., Wyss, M. T., Elsayed, M., Stobart, J., Gutierrez, R., von Faber-Castell, A., et al. (2016). In vivo evidence for a lactate gradient from astrocytes to neurons. Cell Metab. 23, 94-102. doi: 10.1016/j.cmet.2015.10.010

Magistretti, P. J. (2006). Neuron-glia metabolic coupling and plasticity. J. Exp. Biol. 209, 2304-2311. doi: 10.1242/jeb.02208

Magistretti, P. J., and Allaman, I. (2015). A cellular perspective on brain energy metabolism and functional imaging. Neuron 86, 883-901. doi: 10.1016/j. neuron.2015.03.035

Mason, S. (2017). Lactate shuttles in neuroenergetics-homeostasis, allostasis and beyond. Front. Neurosci. 11:43. doi: 10.3389/fnins.2017.00043

Mathiisen, T. M., Lehre, K. P., Danbolt, N. C., and Ottersen, O. P. (2010). The perivascular astroglial sheath provides a complete covering of the brain microvessels: an electron microscopic 3D reconstruction. Glia 58, 1094-1103. doi: 10.1002/glia.20990

Mazuel, L., Blanc, J., Repond, C., Bouchaud, V., Raffard, G., Déglon, N., et al. (2017). A neuronal MCT2 knockdown in the rat somatosensory cortex reduces both the NMR lactate signal and the BOLD response during whisker stimulation. PLoS One 12:e0174990. doi: 10.1371/journal.pone.0174990

Mink, J. W., Blumenschine, R. J., and Adams, D. B. (1981). Ratio of central nervous system to body metabolism in vertebrates: its constancy and functional basis. Am. J. Physiol. 241, R203-R212. doi: 10.1152/ajpregu.1981.241.3.R203

Mitchell, P., Liew, G., Gopinath, B., and Wong, T. Y. (2018). Age-related macular degeneration. Lancet 392, 1147-1159. doi: 10.1016/S0140-6736(18)31550-2

Mongeon, R., Venkatachalam, V., and Yellen, G. (2016). Cytosolic NADH-NAD(+) redox visualized in brain slices by two-photon fluorescence lifetime biosensor imaging. Antioxid. Redox Signal. 25, 553-563. doi: 10.1089/ars.2015.6593

Namba, T., Nardelli, J., Gressens, P., and Huttner, W. B. (2020). Metabolic regulation of neocortical expansion in development and evolution. Neuron 109, 408-419. doi: 10.1016/j.neuron.2020.11.014

Narayan, D. S., Chidlow, G., Wood, J. P., and Casson, R. J. (2017). Glucose metabolism in mammalian photoreceptor inner and outer segments. Clin. Exp. Ophthalmol. 45, 730-741. doi: 10.1111/ceo.12952

Narayan, D. S., Wood, J. P. M., Chidlow, G., and Casson, R. J. (2016). A review of the mechanisms of cone degeneration in retinitis pigmentosa. Acta Ophthalmol. 94, 748-754. doi: 10.1111/aos.13141

Ng, S. K., Wood, J. P. M., Chidlow, G., Han, G., Kittipassorn, T., Peet, D. J., et al. (2015). Cancer-like metabolism of the mammalian retina. Clin. Exp. Ophthalmol. 43, 367-376. doi: 10.1111/ceo.12462

Nortley, R., and Attwell, D. (2017). Control of brain energy supply by astrocytes. Curr. Opin. Neurobiol. 47, 80-85. doi: 10.1016/j.conb.2017.09.012

Oberheim, N. A., Wang, X., Goldman, S., and Nedergaard, M. (2006). Astrocytic complexity distinguishes the human brain. Trends Neurosci. 29, 547-553. doi: 10.1016/j.tins.2006.08.004

O’Brien, J., Kla, K. M., Hopkins, I. B., Malecki, E. A., and McKenna, M. C. (2007). Kinetic parameters and lactate dehydrogenase isozyme activities support possible lactate utilization by neurons. Neurochem. Res. 32, 597-607. doi: 10 . 1007/s11064-006-9132-9

Okawa, H., Sampath, A. P., Laughlin, S. B., and Fain, G. L. (2008). ATP consumption by mammalian rod photoreceptors in darkness and in light. Curr. Biol. 18, 1917-1921. doi: 10.1016/j.cub.2008.10.029

Park, K. S., Xu, C. L., Cui, X., and Tsang, S. H. (2018). Reprogramming the metabolome rescues retinal degeneration. Cell. Mol. Life Sci. 75, 1559-1566. doi: 10.1007/s00018-018-2744-9

Pellerin, L., Bergersen, L. H., Halestrap, A. P., and Pierre, K. (2005). Cellular and subcellular distribution of monocarboxylate transporters in cultured brain cells and in the adult brain. J. Neurosci. Res. 79, 55-64. doi: 10.1002/jnr.2 0307

Pellerin, L., and Magistretti, P. J. (1994). Glutamate uptake into astrocytes stimulates aerobic glycolysis: a mechanism coupling neuronal activity to glucose utilization. Proc. Natl. Acad. Sci. U.S.A. 91, 10625-10629. doi: 10.1073/pnas.91. 22.10625
Pellerin, L., Pellegri, G., Bittar, P. G., Charnay, Y., Bouras, C., Martin, J. L., et al. (1998). Evidence supporting the existence of an activity-dependent astrocyteneuron lactate shuttle. Dev. Neurosci. 20, 291-299. doi: 10.1159/000017324

Petit, L., Ma, S., Cipi, J., Cheng, S.-Y., Zieger, M., Hay, N., et al. (2018). Aerobic glycolysis is essential for normal rod function and controls secondary cone death in retinitis pigmentosa. Cell Rep. 23, 2629-2642. doi: 10.1016/j.celrep. 2018.04.111

Rangaraju, V., Calloway, N., and Ryan, T. A. (2014). Activity-driven local ATP synthesis is required for synaptic function. Cell 156, 825-835. doi: 10.1016/j. cell.2013.12.042

Reyes-Reveles, J., Dhingra, A., Alexander, D., Bragin, A., Philp, N. J., and BoeszeBattaglia, K. (2017). Phagocytosis-dependent ketogenesis in retinal pigment epithelium. J. Biol. Chem. 292, 8038-8047. doi: 10.1074/jbc.M116.770784

Ripps, H. (2002). Cell death in retinitis pigmentosa: gap junctions and the 'bystander' effect. Exp. Eye Res. 74, 327-336. doi: 10.1006/exer.2002.1155

Ruminot, I., Schmälzle, J., Leyton, B., Barros, L. F., and Deitmer, J. W. (2019). Tight coupling of astrocyte energy metabolism to synaptic activity revealed by genetically encoded FRET nanosensors in hippocampal tissue. J. Cereb. Blood Flow Metab. 39, 513-523. doi: 10.1177/0271678X17737012

Sokoloff, L. (1977). Relation between physiological function and energy metabolism in the central nervous system. J. Neurochem. 29, 13-26. doi: 10. 1111/j.1471-4159.1977.tb03919.x

Sotelo-Hitschfeld, T., Niemeyer, M. I., Mächler, P., Ruminot, I., Lerchundi, R., Wyss, M. T., et al. (2015). Channel-mediated lactate release by K?-stimulated astrocytes. J. Neurosci. 35, 4168-4178. doi: 10.1523/JNEUROSCI.5036-14.2015

Supplie, L. M., Düking, T., Campbell, G., Diaz, F., Moraes, C. T., Götz, M., et al. (2017). Respiration-Deficient astrocytes survive as glycolytic cells in vivo. J. Neurosci. 37, 4231-4242. doi: 10.1523/JNEUROSCI.0756-16.2017

Tang, B. L. (2018). Brain activity-induced neuronal glucose uptake/glycolysis: is the lactate shuttle not required? Brain Res. Bull. 137, 225-228. doi: 10.1016/j. brainresbull.2017.12.010

Terluk, M. R., Ebeling, M. C., Fisher, C. R., Kapphahn, R. J., Yuan, C., Kartha, R. V., et al. (2019). N-Acetyl-L-cysteine protects human retinal pigment epithelial cells from oxidative damage: implications for age-related macular degeneration. Oxid. Med. Cell. Longev. 2019:5174957. doi: 10.1155/2019/5174957

Terluk, M. R., Kapphahn, R. J., Soukup, L. M., Gong, H., Gallardo, C., Montezuma, S. R., et al. (2015). Investigating mitochondria as a target for treating age-related macular degeneration. J. Neurosci. 35, 7304-7311. doi: 10.1523/JNEUROSCI. 0190-15.2015

van Hall, G., Strømstad, M., Rasmussen, P., Jans, O., Zaar, M., Gam, C., et al. (2009). Blood lactate is an important energy source for the human brain. J. Cereb. Blood Flow Metab. 29, 1121-1129. doi: 10.1038/jcbfm.2009.35

Vander Heiden, M. G., Cantley, L. C., and Thompson, C. B. (2009). Understanding the Warburg effect: the metabolic requirements of cell proliferation. Science 324, 1029-1033. doi: 10.1126/science.1160809

Verstreken, P., Ly, C. V., Venken, K. J. T., Koh, T.-W., Zhou, Y., and Bellen, H. J. (2005). Synaptic mitochondria are critical for mobilization of reserve pool vesicles at Drosophila neuromuscular junctions. Neuron 47, 365-378. doi: 10.1016/j.neuron.2005.06.018

Veys, K., Fan, Z., Ghobrial, M., Bouché, A., García-Caballero, M., Vriens, K., et al. (2020). Role of the GLUT1 glucose transporter in postnatal CNS angiogenesis and blood-brain barrier integrity. Circ. Res. 127, 466-482. doi: 10.1161/CIRCRESAHA.119.316463

Volkenhoff, A., Weiler, A., Letzel, M., Stehling, M., Klämbt, C., and Schirmeier, S. (2015). Glial glycolysis is essential for neuronal survival in Drosophila. Cell Metab. 22, 437-447. doi: 10.1016/j.cmet.2015.07.006

Wang, W., Fernandez de Castro, J., Vukmanic, E., Zhou, L., Emery, D., Demarco, P. J., et al. (2011). Selective rod degeneration and partial cone inactivation characterize an iodoacetic acid model of Swine retinal degeneration. Invest. Opthalmol. Vis. Sci. 52, 7917-7923. doi: 10.1167/iovs.11-7849

Wang, W., Kini, A., Wang, Y., Liu, T., Chen, Y., Vukmanic, E., et al. (2019). Metabolic deregulation of the blood-outer retinal barrier in retinitis pigmentosa. Cell Rep. 28, 1323-1334.e4. doi: 10.1016/j.celrep.2019.06.093

Wang, W., Lee, S. J., Scott, P. A., Lu, X., Emery, D., Liu, Y., et al. (2016). Two-Step reactivation of dormant cones in retinitis pigmentosa. Cell Rep. 15, 372-385. doi: 10.1016/j.celrep.2016.03.022

Warburg, O. (1956). On the origin of cancer cells. Science 123, 309-314. doi: 10.1126/science.123.3191.309 
Warburg, O., Posener, K., and Negelein, E. (1924). Ueber den Stoffwechsel der tumoren. Biochem. Z. 152, 309-344.

Ward, P. S., and Thompson, C. B. (2012). Metabolic reprogramming: a cancer hallmark even warburg did not anticipate. Cancer Cell 21, 297-308. doi: 10. 1016/j.ccr.2012.02.014

Watanabe, T., Matsushima, S., Okazaki, M., Nagamatsu, S., Hirosawa, K., Uchimura, H., et al. (1996). Localization and ontogeny of GLUT3 expression in the rat retina. Brain Res. Dev. Brain Res. 94, 60-66. doi: 10.1016/0165-3806(96) 00044-2

Wong-Riley, M. T. T. (2010). Energy metabolism of the visual system. Eye Brain 2, 99-116. doi: 10.2147/EB.S9078

Wyss, M. T., Jolivet, R., Buck, A., Magistretti, P. J., and Weber, B. (2011). In vivo evidence for lactate as a neuronal energy source. J. Neurosci. 31, 7477-7485. doi: 10.1523/JNEUROSCI.0415-11.2011

Young, R. W. (1967). The renewal of photoreceptor cell outer segments. J. Cell Biol. 33, 61-72. doi: 10.1083/jcb.33.1.61

Young, R. W. (1971). The renewal of rod and cone outer segments in the rhesus monkey. J. Cell Biol. 49, 303-318. doi: 10.1083/jcb.49.2.303

Yuan, Y., Huo, H., and Fang, T. (2018). Effects of metabolic energy on synaptic transmission and dendritic integration in pyramidal neurons. Front. Comput. Neurosci. 12:79. doi: 10.3389/fncom.2018. 00079

Zhang, Y., Chen, K., Sloan, S. A., Bennett, M. L., Scholze, A. R., O'Keeffe, S., et al. (2014). An RNA-sequencing transcriptome and splicing database of glia, neurons, and vascular cells of the cerebral cortex. J. Neurosci. 34, 11929-11947. doi: 10.1523/JNEUROSCI.1860-14.2014

Zimmer, E. R., Parent, M. J., Souza, D. G., Leuzy, A., Lecrux, C., Kim, H.-I., et al. (2017). 18FFDG PET signal is driven by astroglial glutamate transport. Nat. Neurosci. 20, 393-395. doi: 10.1038/nn.4492

Conflict of Interest: The authors declare that the research was conducted in the absence of any commercial or financial relationships that could be construed as a potential conflict of interest.

Copyright (C) 2021 Viegas and Neuhauss. This is an open-access article distributed under the terms of the Creative Commons Attribution License (CC BY). The use, distribution or reproduction in other forums is permitted, provided the original author(s) and the copyright owner(s) are credited and that the original publication in this journal is cited, in accordance with accepted academic practice. No use, distribution or reproduction is permitted which does not comply with these terms. 